\title{
Fanconi anaemia in black South African patients heterozygous for the FANCG c.637-643delTACCGCC founder mutation
}

T Wainstein, ${ }^{1}$ MSc (Med); R Kerr, ${ }^{1}$ PhD; C L Mitchell, ${ }^{1}$ MSc (Med); S Madaree, ${ }^{1}$ BSc (Hons); F B Essop, ${ }^{1}$ MSc (Med); E Vorster, ${ }^{1}$ BSc (Hons); R Wainwright, ${ }^{2} \mathrm{MB}$ BCh; J Poole, ${ }^{3} \mathrm{MB} \mathrm{BCh}$; A Krause, ${ }^{1} \mathrm{MB} \mathrm{BCh}, \mathrm{PhD}$

${ }^{1}$ Division of Human Genetics, School of Pathology, National Health Laboratory Service and Faculty of Health Sciences, University of the Witwatersrand, Johannesburg, South Africa

${ }^{2}$ Division of Haematology and Oncology, Department of Paediatrics, Chris Hani Baragwanath Academic Hospital and Faculty of Health Sciences, University of the Witwatersand, Johannesburg, South Africa

${ }^{3}$ Division of Haematology and Oncology, Department of Paediatrics, Charlotte Maxeke Johannesburg Academic Hospital and Faculty of Health Sciences, University of the Witwatersrand, Johannesburg, South Africa

Corresponding author: T Wainstein (tasha.wainstein@nhls.ac.za)

Background. Fanconi anaemia (FA) is an autosomal recessive, genetically heterogeneous disorder, characterised by interstrand crosslinkinduced chromosome breaks, congenital abnormalities and predisposition to malignancies. It has a prevalence of about 1/40 000 in black South Africans (SAs). A founder mutation in the FANCG gene occurs in the homozygous state in $77.5 \%$ of southern African blacks.

Objective. To locate additional pathogenic mutations in the FANCG gene of black FA patients who were heterozygous for the founder mutation. Methods. Further mutation analysis of the FANCG gene was undertaken in 7 patients clinically suspected of having FA. The parents of two of the patients were tested for the presence of the founder mutation to determine true heterozygosity in the patients. To clarify whether or not previously unreported variants were pathogenic, 58 random black SA individuals were screened.

Results. Three novel single base pair deletions, resulting in frameshift mutations (c.247delA, c.179delT and c.899delT) were identified in 3/7 patients. A fourth patient was found to have a single base substitution resulting in a splice site mutation (c.1636+1G>A). The remaining three patients were not found to harbour any pathogenic mutations. Two non-pathogenic variants were also identified among the seven patients. Conclusion. The results of this small sample suggest that a second common mutation in the FANCG gene is unlikely in this population. However, FANCG sequencing should be performed on patients heterozygous for the common founder mutation to attempt to confirm their diagnosis.

S Afr Med J 2013;103(12 Suppl 1):970-973. DOI:10.7196/SAMJ.7215 


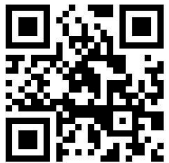

Fanconi anaemia (FA) is characterised by interstrand crosslink-induced chromosome breaks ${ }^{[1]}$ congenital abnormalities (including radial-ray aplasia, heart and kidney malformations), ${ }^{[2]}$ bone marrow failure, ${ }^{[3]}$ and predisposition to haematological and solid malignancies. ${ }^{[4]}$ The condition is rare, with a worldwide incidence of about $1 / 300000^{[5]}$ and a minimum carrier frequency of $1 / 300{ }^{[6]}$

FA is a highly heterogeneous condition, both clinically and genetically, arising from mutations in 1 of at least 15 genes. ${ }^{[-9]}$ All forms of FA are inherited as autosomal recessive conditions except for $F A N C B$, which is an X-linked disorder. Loss-of-function mutations in the genes that underlie each of the FA complementation groups have been found directly or indirectly to play a role in disrupting the DNA repair pathway of cells. ${ }^{[7]}$

Since the 1970s, FA has been subject to investigation in the Division of Human Genetics, National Health Laboratory Service and the University of the Witwatersrand, South Africa (SA). Initially, research focussed on the understanding of both the clinical manifestations and the underlying genetic aetiology of FA in the SA white Afrikaans population. Rosendorff $\mathrm{et}$ al ${ }^{[10]}$ estimated a minimum birth incidence of FA in white, Afrikaans-speaking individuals to be $1 / 22000$ with a carrier frequency of $1 / 77$. It was postulated at the time that this increased incidence could be attributed to a founder effect. This hypothesis was later proved to be correct when Tipping et al ${ }^{[11]}$ identified multiple founder mutations in the FANCA gene in this population.

Similar investigations have been undertaken in the black SA population. Initial reports seemed to suggest FA in black individuals was very rare.$^{[12]}$ However, Macdougall et al ${ }^{[13]}$ estimated the incidence of FA in this population as 1/476 000 in a case-based ascertainment study. More recently, gene frequency studies have shown that FA has a carrier frequency of 1/100 in black SA patients and therefore an expected birth incidence of $1 / 40000{ }^{[14] .}$ The discrepancy between these two incidence rates is most likely due to an under-diagnosis of FA at local and regional healthcare facilities. FA in this population has been found to be caused largely by mutations in the FANCG gene. ${ }^{[14]}$

Evidence further suggested that the clinical manifestations of FA in this population were similar to those in other ethnic groups. ${ }^{[12]}$ This finding was further confirmed in a comparative study of FA in various SA ethnic groups. ${ }^{[13]}$ Newer studies in black patients with known FANCG mutations have identified both similarities and differences with regards to the phenotypic manifestations of FA. One study showed that black individuals with FANCG mutations had more features in common with a group of other FANCG patients than with a group of FANCA patients. ${ }^{[15]}$ An additional study indicated that there were no particular features of the FANCG phenotype in black patients that would allow prediction regarding the prognosis of affected individuals. ${ }^{[16]}$

The FANCG gene maps to chromosome 9p13, is $6 \mathrm{~kb}$ in length comprising 14 exons and encoding a $2.5 \mathrm{~kb}$ mRNA transcript ${ }^{[17]}$ This is translated into a 622 amino acid polypeptide with a molecular weight of $68 \mathrm{kDa} \cdot{ }^{[18]}$ Mutations in the FANCG gene account for $8.8 \%$ of FA cases worldwide. ${ }^{[19]}$

A common 7 base pair deletion founder mutation (c.637643delTACCGCC) in the FANCG gene has been found in the homozygous state in $77.5 \%$ (31/40) of South African black patients and in a further $5 \%(2 / 40)$ of patients in the heterozygous state. It has also been found in black patients from Swaziland, Mozambique and Malawi. ${ }^{[14]}$

A number of black SA individuals, strongly suspected of having FA based on their clinical features, have now been found to be heterozygous for the c.637-643delTACCGCC founder mutation. There are limited diagnostic services available locally, and a reliable cytogenetic confirmation of FA is not always possible. This situation has resulted in an increased reliance on clinical and molecular diagnosis in these patients as opposed to chromosome fragility testing.

\section{Objective}

To undertake molecular testing to identify the second FA-causing mutation in patients who were heterozygous for the founder mutation in the FANCG gene, as well as to determine whether or not other FANCG founder mutations exist in this population.

\section{Methods}

Seven black SA patients, strongly suspected clinically to have FA and heterozygous for the c.637-643delTACCGCC mutation, were investigated. Of the seven patients, four also underwent cytogenetic analysis. The parents of two of the patients were available and tested for the presence of the founder mutation to determine true heterozygosity and exclude gene conversion in the probands.

DNA sequence analysis for all 14 exons of the FANCG gene, using the ABI3130xl genetic analyser (Applied Biosystems), was undertaken. Peripheral blood was used as the source of DNA for the molecular analysis of these patients. The primers used in this study were modifications (Table 2, provided online) of those outlined previously. ${ }^{[20]}$ Sequencing results were compared with normal reference sequences (accessed September 2009, Ensembl) using the SeqMan application of DNAstar (Lasergene).

For variants where the pathogenicity was unclear, 58 random black SA individuals were screened using restriction enzyme analysis to determine if these changes were polymorphic. These control samples were obtained from the sample bank of the Division of Human Genetics. If a variant is identified in the normal population at polymorphic frequencies, it is unlikely to be the cause of disease in a patient.

Ethics clearance was obtained for this study from the Human Research Ethics Committee (HREC), University of the Witwatersrand.

\section{Results}

Screening of the entire coding region as well as the flanking intronic regions of the FANCG gene was undertaken in the seven patients. A second disease-causing mutation was found in $4 / 7$ patients (FA82, FA121, FA199 and FA465) (Table 1).

Although a second pathogenic mutation was not identified in the FANCG gene of the remaining three patients (FA189, FA312 and FA402), the presence of large deletions, insertions or rearrangements cannot be excluded as the causal mutations in these patients. Unfortunately, dosage analysis by multiplex ligation-dependent probe amplification (MLPA) is not commercially available for the FANCG gene. Cost has prevented the development of an in-house assay.

The parents of patients FA189 and FA312 were tested for the common founder mutation (c.637-643delTACCGCC) and in both cases only one parent was found to be heterozygous for this mutation. Patient FA402 was lost to follow-up and therefore molecular analysis of the patient's parents was not performed. Sequencing of the parents' FANCG genes for non-founder mutations has not been performed.

\section{Frameshift mutations}

The exonic single base deletion mutations in patients FA82, FA121 and FA199 all resulted in frameshift mutations. These mutations lead to premature stop codons and to the production of truncated and non-functional proteins. In FA82, the new open reading frame erroneously incorporated an additional 17 amino acids, FA121 had an additional 6 amino acids and FA199 had an additional 11 amino acids before the new premature stop codon. 
Table 1. Mutation analysis of the FANCG gene and cytogenetic tests on black FA patients heterozygous for c.637-643delTACCGCC $(N=7)$

\begin{tabular}{|c|c|c|c|c|}
\hline Patient & Position & Genotype & Effect & Cytogenetic result \\
\hline FA82 & Exon 3 & c.247delA (p.Ser83Alafs ${ }^{\star} 17$ ) & Frameshift mutation (pathogenic) & Positive \\
\hline FA121 & Exon 7 & c.899delT (p.Leu300Argfs $\left.{ }^{\star} 6\right)$ & Frameshift mutation (pathogenic) & Positive \\
\hline FA189 & Exon 2 & c.208G>A (p.Glu70Lys) & Polymorphism (non-pathogenic) & Positive \\
\hline FA199 & Exon 3 & c.179delT (p.Leu60Profs $\left.{ }^{\star} 11\right)$ & Frameshift mutation (pathogenic) & Not done \\
\hline FA312 & - & - & No variation observed & Not done \\
\hline FA402 & - & - & No variation observed & Unsuccessful \\
\hline \multirow[t]{3}{*}{ FA465 } & Intron 12 & c. $1636+1 \mathrm{G}>\mathrm{A}$ & Splice donor site mutation (pathogenic) & \\
\hline & Intron 4 & c. $510+55 \mathrm{C}>\mathrm{T}$ & Polymorphism (non-pathogenic) & Not done \\
\hline & Exon 7 & c.890C>T (p.Thr297Ile) & Polymorphism (non-pathogenic) & \\
\hline
\end{tabular}

\section{Splice mutations}

A novel c. $1636+1 \mathrm{G}>\mathrm{A}$ mutation was discovered in intron 12 in the FANCG gene of patient FA465. This position is part of the splice donor site, and mutations here are predicted to lead to readthrough during mRNA processing. At the protein level, the readthrough becomes translated into 5 extra amino acids followed by a premature stop codon.

\section{Non-pathogenic mutations}

Non-pathogenic mutations were identified in patients FA189 and FA465 (Table 1).

In subject FA189, a novel non-synonymous c.208G $>A$ variant was identified in exon 2. This novel variant was screened for in a group of 58 random black individuals from the SA population by restriction enzyme analysis. It was found that the $G$ allele occurs at a frequency of 0.85 (standard deviation (SD) \pm 0.03 ), while the A allele was found at a frequency of $0.15(\mathrm{SD} \pm 0.03)$ in the normal population.

The variants identified in subject FA465 (i.e. c. $510+55 \mathrm{C}>\mathrm{T}$ and c. $890 \mathrm{C}>\mathrm{T}$ ) were classified as non-pathogenic. Neither variant was previously reported in $\mathrm{dbSNP}^{[21]}$ Ensembl $^{[22]}$ or the FA mutation database. ${ }^{[23]}$ The position of the $c .510+55 \mathrm{C}>\mathrm{T}$ variant within an intron made it unlikely to be pathogenic. HapMap data indicated that the allele frequency of the $\mathrm{T}$ allele in the c.890C $>\mathrm{T}$ variant is 0.125 in a sub-Saharan African population while that of the $\mathrm{C}$ allele is 0.875 . The variant is therefore considered polymorphic in this population.

\section{Discussion}

A second disease-causing mutation was identified in 4/7 FA patients, heterozygous for the common c.637-647delTACCGCC mutation in the FANCG gene. Three novel single base pair deletions resulting in frameshift mutations (c.247delA, c.179delT and c.899delT) were identified and a novel single base substitution, resulting in a splice donor site mutation (c.1636+1G>A), was also found in this study.

Patient FA189 was shown to have a novel, coding, non-synonymous, single-nucleotide polymorphism (SNP) (c.208G>A). As the A allele is present in $15 \%$ of the normal black population, this variant is predicted to be non-pathogenic. However, non-pathogenic variants identified in two of the patients (FA189 and FA465) could be used as linked markers for family studies to determine carrier status and for prenatal diagnosis. This is particularly true in the case of FA189, where a second mutation was not identified. No variants useful for linked marker analysis were identified in patients FA312 or FA402.

In the three patients in whom the second pathogenic mutation was not identified, large deletions, insertions or rearrangements in the FANCG gene cannot be excluded as the cause of FA. Also, cryptic splice sites or mutations in control elements lying deep within an intron would have been missed using the approach of this study. True heterozygosity for the founder mutation was confirmed in $2 / 3$ patients by testing the parents. Somatic gene conversion of the rare mutation to normal cannot be excluded as DNA from peripheral blood was tested and the DNA from the parent with the non-founder mutation was not sequenced.

Considering that only private mutations were identified in this study, it is possible to conclude that a second common pathogenic mutation in this population is unlikely to be found in the FANCG gene. SNP analysis may be useful in tracking the high-risk chromosome in families of patients where the second disease-causing mutation has not been identified, assuming the diagnosis is correct.

\section{Conclusion}

Molecular diagnosis of FA in the SA context is unique in terms of approach: there are three large population groups in SA and each has a high incidence of FA. These are the black, Ashkenazi Jewish and Afrikaner populations. Founder mutations have been identified in different FA genes for each of these populations. ${ }^{[11,14,24]}$ As such, molecular genetic diagnosis for FA in SA is specifically targeted depending on ethnicity (self-reported). In view of the limited availability of diagnostic chromosomal breakage studies, an alternative molecular diagnostic approach is favoured in patients clinically suspected to have FA. For rare individuals with a suspected diagnosis of FA not falling into one of these population groups, a testing approach taking into account the marked genetic heterogeneity would need to be taken.

The first-line test for individuals from the black population should be for the common founder mutation. Full sequencing of the FANCG gene is then warranted in black FA patients who are found to be heterozygous for the FANCG founder mutation as a second mutation can sometimes be identified. It is additionally recommended that the parents of such individuals be tested as well to exclude the possibility of gene conversion. The use of deletion/duplication analysis for FANCG still requires evaluation.

In patients in whom the FANCG founder mutation is not identified, FA is probably due to a gene other than FANCG. Future research could help to determine which other mutations in FA genes contribute to the remaining unidentified proportion of FA in the black population.

Acknowledgments. The authors wish to acknowledge the clinicians who referred these patients to the Department and the NHLS Research Trust (Grant no. 94075) for funding. 


\section{References}

1. Grompe M, D’Andrea AD. Fanconi anaemia and DNA repair. Hum Mol Genet 2001;10(20)2253-2259. [http://dx.doi.org/10.1093/hmg/10.20.2253]

. De Kerviler E, Guermazi A, Zagdanski AM, et al. The clinical and radiological features of Fanconi anaemia. Clin Radiol 2000;55(5):340-345. [http://dx.doi.org/10.1053/crad.1999.0445]

3. Alter BP. Hematological abnormalities in Fanconi anaemia. Blood 1995;85:1148-1149.

4. Alter BP. Fanconis anaemia and malignancies. Am J Hematol 1996(2);53:99-110. [http://dx.doi. org/10.1002/(SICI)1096-8652(199610)53:2<99::AID-AJH7>3.0.CO;2-Z]

5. Rogatko A, Auerbach AD. Segregation analysis with uncertain ascertainment: Application to Fanconi anaemia. Am J Hum Genet 1988;42:889-897.

6. Swift M. Fanconi's anaemia in the genetics of neoplasia. Nature 1971;230(5293):370-373. [http:// dx.doi.org/10.1038/230370a0]

7. De Winter JP, Joenje H. The genetic and molecular basis of Fanconi anaemia. Mutat Res 2009;668(12):11-19. [http://dx.doi.org/10.1016/j.mrfmmm.2008.11.004]

8. Vaz F, Hannenberg H, Schuster B, et al. Mutation of the RAD51C gene in Fanconi-like disorder. Nat Genet 2010;42(5):406-411. [http://dx.doi.org/10.1038/ng.570]

. Kim Y, Lach FP, Desetty R, et al. Mutations of the SLX4 gene in Fanconi anaemia. Nat Genet 2011;43(2):142-147. [[http://dx.doi.org/10.1038/ng.750]

10. Rosendorff J, Bernstein R, Macdougall L, Jenkins T. Fanconi anemia: Another disease of unusually high prevalence in the Afrikaans population of South Africa. Am J Med Genet 1987;27:793-797.

11. Tipping AJ, Pearson T, Morgan NV, et al. Molecular and genealogical evidence for a founder effect in Fanconi anaemia families of the Afrikaner population of South Africa. PNAS 2001;98(10):5734-5739. [http://dx.doi.org/10.1073/pnas.091402398]

12. Macdougall L, Greef MC, Rosendorff J, et al. Fanconi anemia in black African children. Am J Med Genet 1990;36(4):408-413. [http://dx.doi.org/10.1002/ajmg.1320360408]

13. Macdougall L, Rosendorff J, Poole JE, et al. Comparative study of Fanconi anemia in children of different ethnic origin in South Africa. Am J Med Genet 1994;52(3):279-284. [http://dx.doi.
ond of different ethnic origin in
org/10.1002/ajmg.1320520306

14. Morgan NV, Essop FB, Demuth I, et al. A common Fanconi anaemia mutation in black populations of sub-Saharan Africa. Blood 2005;105(9):3542-3544. [http://dx.doi.org/10.1182/blood-2004-10-3968]
15. Haw, T. FANCG 637-643 deletion mutation: Frequency in black patients with acute myeloid leukaemia or aplastic anaemia and the clinical phenotype of homozygotes. Unpublished dissertation. Johannesburg: University of the Witwatersrand, 2005 .

16. Feben, C. Phenotypic consequences in black South African Fanconi anaemia patients homozygous for a FANCG 637-643 deletion mutation. Unpublished dissertation. Johannesburg: University of the Witwatersrand, 2012.

17. Liu N, Lamerdin JE, Tucker JD, et al. The human XRCC9 gene corrects chromosomal instability and mutagen sensitivities in CHO UV40 cells. PNAS 1997;94(17):9232-9237.

18. De Winter JP, Waisfisz Q, Rooimans MA, et al. The Fanconi anaemia group G gene FANCG is identical with XRCC9. Nat Genet 1998;20(3):281-283.

19. Grompe M, D’Andrea AD. The Fanconi anaemia/BRCA pathway. Nat Rev Canc 2003:3(1):23-34.

20. Auerbach AD, Greenbaum J, Pujara K, et al. Spectrum of sequence variation in the FANCG gene: An International Fanconi Anaemia Registry (IFAR) study. Hum Mutat 2003;21:158-168. [http://dx.doi.

org $/ 10.1002 /$ humu. 10166]
21. Kitts A, Sherry S. The Single Nucleotide Polymorphism Database (dbSNP) of Nucleotide Sequence Variation. In: The NCBI Handbook. McEntyre J, Ostell J, eds. Bethesda, MD: US National Center for Biotechnology Information, 2002 http://www.ncbi.nlm.nih.gov/books/NBK21088 (accessed 15 September 2009).

22. Hubbard TJP, Aken BL, Beal K, et al. Ensembl 2007. Nucleic Acids Res 2007;35(Suppl 1):D610-D617. [http://dx.doi.org/10.1093/nar/gkl996]

23. International Fanconi Anemia Registry. Fanconi anemia mutation database 1998. http://www. rockefeller.edu/fanconi/ (accessed 14 October 2009).

24. Whitney MA, Saito $\mathrm{H}$ Jakobs $\mathrm{PM}$ et al $\mathrm{A}$ common mutation in the FACC gene causes Fanconi anaemia in Ashkenazi Jews. Nat Genet 1993;4(2):202-205. [http://dx.doi.org/10.1038/ng0693-202]

Accepted 12 August 2013 